\title{
Quantitative evaluation of mask phase defects from through-focus EUV aerial images
}

\author{
Iacopo Mochi ${ }^{1}$, Kenji Yamazoe ${ }^{2}$, Andrew Neureuther ${ }^{2}$, Kenneth A. Goldberg ${ }^{1}$, \\ ${ }^{1}$ Center for X-ray Optics, Lawrence Berkeley National Laboratory, Berkeley, CA 94720 \\ ${ }^{2}$ Department of EECS, University of California, Berkeley, CA 94720
}

Mask defects inspection and imaging is one of the most important issues for any pattern transfer lithography technology. This is especially true for EUV lithography where the wavelength-specific properties of masks and defects necessitate actinic inspection for a faithful prediction of defect printability and repair performance. In this paper we will present a technique to obtain a quantitative characterization of mask phase defects from EUV aerial images. We apply this technique to measure the aerial image phase of native defects on a blank mask, measured with the SEMATECH Berkeley Actinic Inspection Tool (AIT) an EUV zoneplate microscope that operates at Lawrence Berkeley National Laboratory. The measured phase is compared with predictions made from AFM topsurface measurements of those defects.

While amplitude defects are usually easy to recognize and quantify with standard inspection techniques like scanning electron microscopy (SEM), defects or structures that have a phase component can be much more challenging to inspect. A phase defect can originate from the substrate or from any level of the multilayer. In both cases its effect on the reflected field is not directly related to the local topography of the mask surface, but depends on the deformation of the multilayer structure.

Using the AIT, we have previously showed that EUV inspection provides a faithful and reliable way to predict the appearance of mask defect on the printed wafer; but to obtain a complete characterization of the defect we need to evaluate quantitatively its phase component.

While aerial imaging doesn't provide a direct measurement of the phase of the object, this information is encoded in the through focus evolution of the image intensity distribution. Recently we developed a technique that allows us to extract the complex amplitude of EUV mask defects using two aerial images from different focal planes.

The method for the phase reconstruction is derived from the Gerchberg-Saxton (GS) algorithm, an iterative method that can be used to reconstruct phase and amplitude of an object from the intensity distributions in the image and in the pupil plane. The GS algorithm is equivalent to a two-parameter optimization problem and it needs exactly two constraints to be solved, namely two intensity distributions in different focal planes. In some formulations, adding any other constraint would result in an ill posed problem. On the other hand, the solution's stability and convergence time can both be improved using more information. We modified our complex amplitude reconstruction algorithm to use 
an arbitrary number of through focus images (see fig. 1) and we compared its performance with the previous version in terms of convergence speed, robustness and accuracy.

We have demonstrated the phase-reconstruction method on native, mask-blank phase defects and compared the results with phase-predictions made from AFM data collected before and after the multilayer deposition. The method and the current results could be extremely useful for improving the modeling and understanding of native phase defects, their detectability, and their printability.

This work was performed under the auspices of the U.S. Department of Energy by University of California Lawrence Berkeley National Laboratory. This work was funded by SEMATECH through the U.S. Department of Energy under Contract No. DE-AC02$05 \mathrm{CH} 11231$.

\section{DISCLAIMER}

This document was prepared as an account of work sponsored by the United States Government. While this document is believed to contain correct information, neither the United States Government nor any agency thereof, nor The Regents of the University of California, nor any of their employees, makes any warranty, express or implied, or assumes any legal responsibility for the accuracy, completeness, or usefulness of any information, apparatus, product, or process disclosed, or represents that its use would not infringe privately owned rights. Reference herein to any specific commercial product, process, or service by its trade name, trademark, manufacturer, or otherwise, does not necessarily constitute or imply its endorsement, recommendation, or favoring by the United States Government or any agency thereof, or The Regents of the University of California. The views and opinions of authors expressed herein do not necessarily state or reflect those of the United States Government or any agency thereof or The Regents of the University of California. 

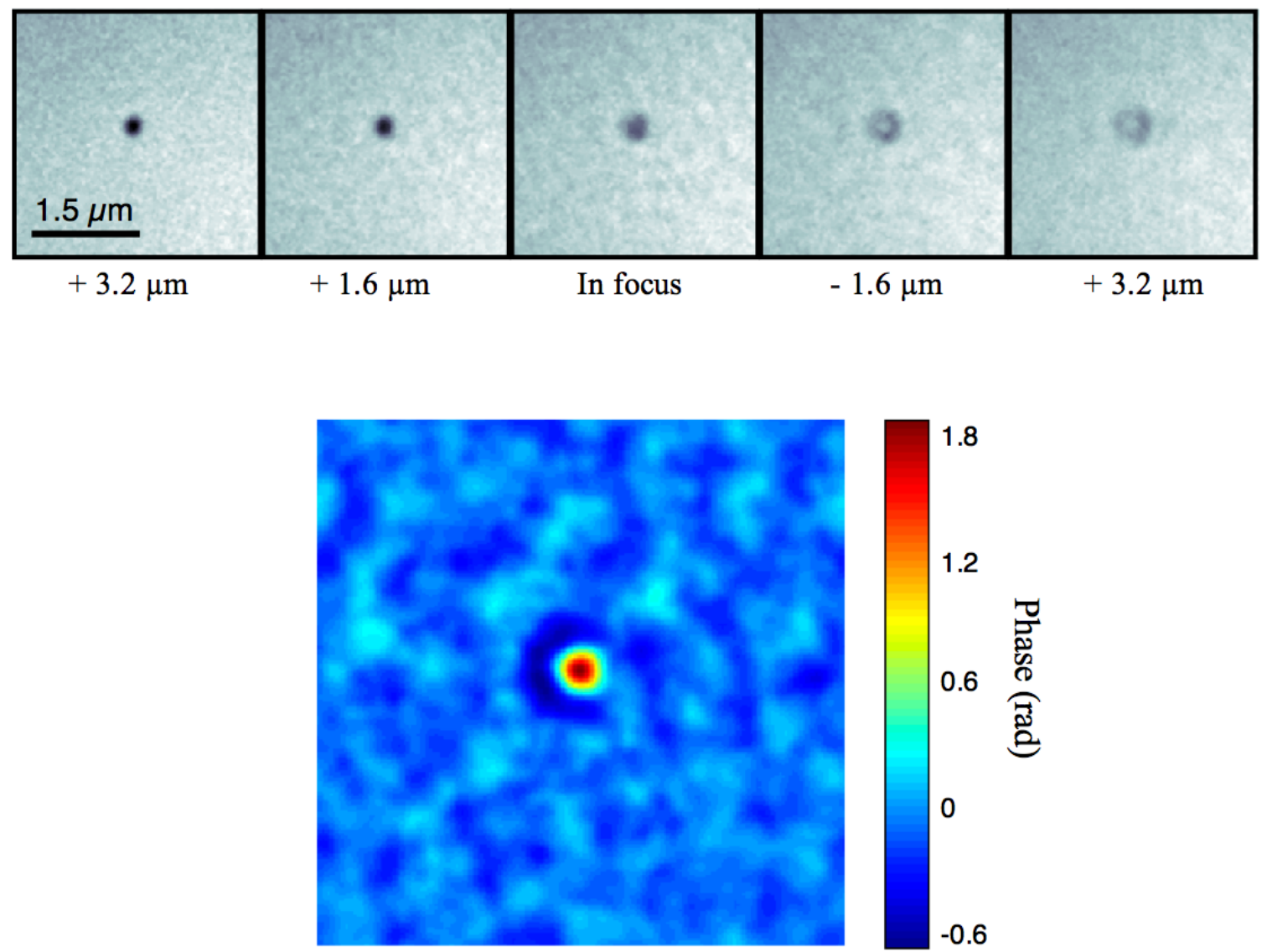

Figure 1. On top we show 5 images of phase defect from an Intel mask blank collected with the AIT at different focal planes, using a NA of 0.0625 and a wavelength of $13.4 \mathrm{~nm}$. We used these images with our phase reconstruction algorithm to extract the complex amplitude of the object. The defect appears to be a pit with a peak phase of $1.8 \mathrm{rad}$. Using a simple single surface approximation model and taking into account the filtering effect of the finite numerical aperture, this would correspond to a pit depth of about $\mathbf{4} \mathbf{~ m m}$ as confirmed by the AFM. 